\title{
Former Editors-in-Chief on Major Developments During Their Time in Office from a Present-Day Perspective
}

\author{
The former editors-in-chief Norbert Szyperski, Peter Mertens, and Wolfgang König \\ outline the major developments in the field of information and communication \\ systems as well as the most important decisions of the WIRTSCHAFTSINFORMATIK \\ journal during their time in office. They discuss these issues from a present-day \\ perspective and additionally highlight the field's and the journal's future challenges.
}

\section{DOI 10.1007/s12599-008-0025-0}

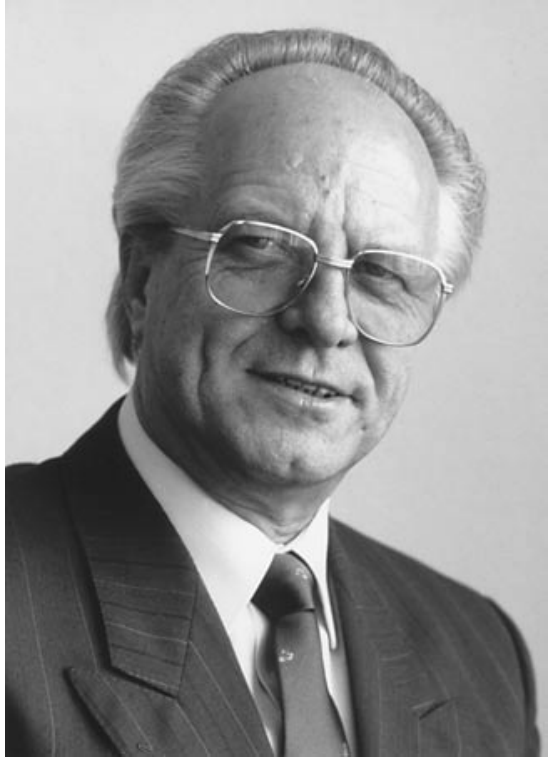

Professor Dr. Dr.h. c. Norbert Szyperski Editor-in-chief from 1971 to 1991 University of Cologne Norbert.Szyperski@uni-koeln.de

BISE: Which developments in the field of information and communication systems did you expect during your time as editorin-chief? Which were the most positive and negative surprises?

This article is also available in German in print and via http://www. wirtschaftsinformatik.de: Szyperski N, Mertens P, König W (2008) Ehemalige Geschäftsführende Herausgeber zu den wesentlichen Entwicklungen ihrer Herausgeberzeit aus heutiger Perspektive. WIRTSCHAFTSINFORMATIK. doi: 10.1007/11576-008-0122-1.
Szyperski: In the beginning of the 1970s, I had strong expectations as regards the development of computer science, the German IT-industry and, of course, particularly of applied computer science and its impacts on IT-usage in national and international business. Development seemed to focus on the integrative handling of highly heterogeneous and large amounts of data as well as on the systems' growing capabilities for - eventually even - open communication. The engineering aspect of designing, realizing, and implementing effective and efficient application systems was about to expand and clearly influenced the world of applications. Thus, the hope was justified that this would also apply to the IT-industry. Our position as regards hardware and basic software sectors was not bad, seen from an international perspective. Thus, there was enough hope to find Germany with its rapidly growing computer science discipline among the leading IT-producers worldwide.

Partly, these expectations were bitterly disappointed in the next two decades, but some were also brilliantly fulfilled. Already in our special issue 2/1982 - on the occasion of mbp's $25^{\text {th }}$ anniversary the missed opportunities became obvious. And this development became more and more disappointing. Computer manufacturers retired completely or began to focus on designing and producing specific hardware components, the broad market of basic software is practically not supplied from Germany anymore. Among other things, this may also have been caused by the unilateral science policy pushing computer science with strong mathematical focus and neglecting computer engineering with its hardware component. And then, of course, there was the German industrial structure: information technology was ultimately seen as a peripheral business, independent IT manufacturers such as Nixdorf became extinct.

So much for the disappointments, let us talk about positive surprises. Applied computer science and its industries developed extraordinarily well in Germany. This applies to business and information systems engineering (BISE) as well as to the various fields of technical applications. The examples of BISE, SAP, ARIS constitute a mere tercet of success bearing Germany's hallmarks with high international impact. Now, many readers may ponder and say: "SAP and IDS Scheer, this is obviously true. But as regards the interdisciplinary field of BISE, is it really clearly visible internationally?" No, obviously not directly, but in fact the slogan should be "BISE inside" - to reveal coherences. While ARIS - to stay with this example - openly shows its BISE background, SAP could not be imagined without it. In the German language, scientific results can only be distributed regionally. If the same methodological and conceptual contents are programmed and coded in a machinereadable way, they lead to internationally acknowledged and respected products. Bellinger once said that German business economics is hardly known internationally although its international impacts are reflected by the success of our globally operating companies.

BISE: Which were the most important decisions or changes for the WIRTSCHAFTSINFORMATIK journal during your time as editor-in-chief?

Szyperski: After the death of Hans Konrad Schuff in 1968, when Paul Schmitz changed to the management of mbp and I 


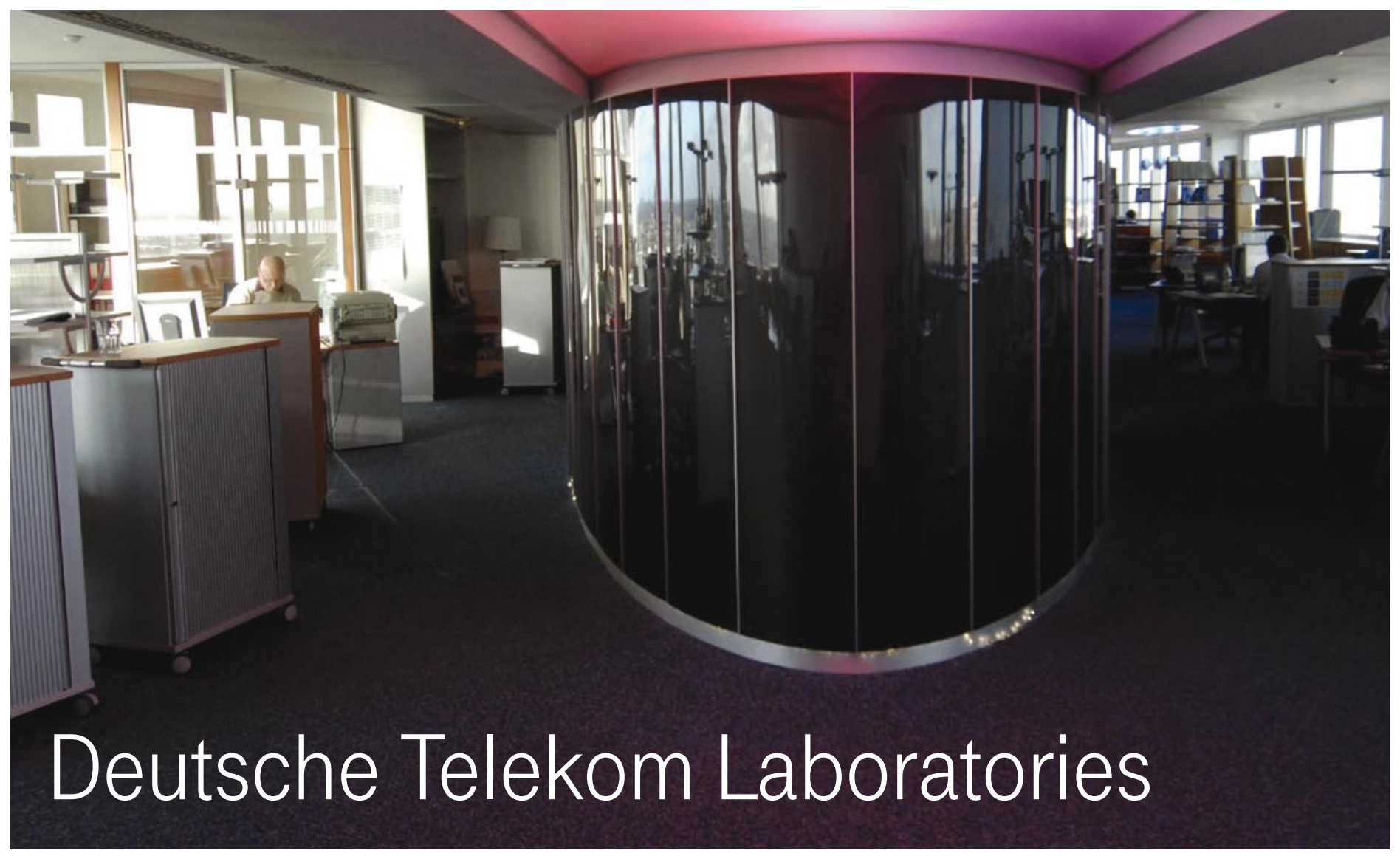

We shape the future

Deutsche Telekom Laboratories is Deutsche Telekom's research and development institute based in Berlin. It is simultaneously a scientific institute organized under private law and associated with the Technische Universität (TU) Berlin. At Deutsche Telekom Laboratories, scientists from across the globe work together with experts from the Group to develop new services and solutions for Deutsche Telekom's customers. Establishing new companies (spin-offs) is another method for making use of research output.

Cooperation with the TU Berlin, other universities and industry partners creates a bridge between business and science in order to turn ideas into marketable innovations as quickly as possible. As part of this, Deutsche Telekom Laboratories focuses on five fields of innovation (5 i):
- Intuitive Usability of services and devices

- Integrated Service Components

- Intelligent Access

- Infrastructure for IT and telecommunications

- Inherent Security

The business and information systems engineering offers useful interdisciplinary approaches for all these areas of innovation. Subject matter includes, for example, modeling, methods and tools for process innovations, agile architectures for information and communication technologies (ICT), technology-oriented management approaches and techno-economic assessments. The aim is to safeguard the economic sustainability of innovations for the Group.

Deutsche Telekom Laboratories is divided into two areas: The Innovation Development Laboratory focuses on market-centric research and development within a timeframe of up to three years.
The basic and technology research of the Strategic Research Laboratory has a long-term focus. Common goal: Deutsche Telekom Laboratories is looking to become one of the world's leading research and development institutions in the field of new ICT.

An institute was set up together with Ben-Gurion University in Beer Sheva, Israel, in 2006. Since 2008, Deutsche Telekom Laboratories has also been represented in Darmstadt. Another project office was opened in the Silicon Valley, United States, in January 2009.

Contact:

Deutsche Telekom Laboratories

Ernst-Reuter-Platz 7

10587 Berlin, Germany

E-mail: wi.laboratories@telekom.de www.laboratories.telekom.com 
became his successor as research director as well as director of the BIFOA in 1970, alongside Erwin Grochla, a decade of close cooperation began. This could already be seen before my official term as editor. Hans Pärli, a co-founder and long-time manager of mbp, talked with us about whether and how to further deal with the journal being so closely associated with mbp. This was the time when electronic data processing became increasingly important in science and society. The former Federal Ministry for Research and Technology launched a support program with respect to data processing. Experts got together and founded the Gesellschaft für Informatik (GI). Paul Schmitz launched the Group of Organization and Data Processing for directors of large-scale data centers. In this spirit of optimism, we decided to continue with the journal elektronische datenverarbeitung (electronic data processing) with a growing focus on data processing applications.

These efforts were accompanied by the pioneering work of the BIFOA, manifested in the first BIFOA memorandum "Application systems for automated data processing. The gap in research and education in the Federal Republic of Germany" (1968). These developments also involved applied computer science research which led to the second data processing program of the Federal Republic and thus offered strong application orientation. This also provided the basis for adapting this journal's name. We agreed on the name "Angewandte Informatik - Applied Informatics" from issue 1/1971 on. Though we had already moved in the direction of our present name WIRTSCHAFTSINFORMATIK/ Business \& Information Systems Engineering by our second BIFOA memorandum "Corporate Information Systems Engineering and Business and Information Systems Engineering as a Necessary Application-Oriented Extension of General Computer Science" (1969) and had taken a stand as regards this subject, it still lasted almost two decades before we were able to launch it with issue $1 / 1990$. This shows that patience is demanded for many things.

The 1970s and 1980s placed other communicative demands on scientific journals than was the case later - not least due to the influence of the Internet. On behalf of the readers, our great editorial staff - managed for many years by Wolf Eicken first and then until today by Ulrich
Hasenkamp - had to point out current news, activities, and interesting details of the IT-markets as well as take on discussions not only about important literature, but also respective events.

As arbitrarily "downloading" papers was not possible at that time, a section was created to which novel research papers could, after an appropriate review, be posted in condensed form and with a corresponding source address. Today it surely would be time to develop a general reference model of papers' scientific contents and especially of gray literature to allow the tracing of the field's actual progress on a constantly updated platform. In this context natural sciences obviously have fewer problems with their theoretical superstructure and their standardized systems.

BISE: How do you assess the developments and changes of that time from a present-day perspective?

Szyperski: By means of the two major decisions for "Applied Informatics" and finally for WIRTSCHAFTSINFORMATIK / Business \& Information Systems Engineering we were able to provide structures which helped unfolding and stabilizing our journal's importance for science and practice in the German-speaking area. The last step found an interim assessment and foundation in the special focus "Business and information systems engineering - A positioning at the beginning of the 1990s" (issue 1/1990). Connecting BISE to the relevant scientific groups, which required some efforts of the editorial board, was also an essential prerequisite for the role of our scientific publication.

Thus, I can only appreciate the new plans for the advancement of WIRTSCHAFTSINFORMATIK / Business \& Information Systems Engineering towards internationalization and practical relevance. I wish the driving promoters continued success and the many BISE community members the necessary creative and systematic force to continue filling the issues with interesting contents.

BISE: Which will be the big challenges of BISE as a scientific discipline in the next years or decades?

Szyperski: BISE will not be able to avoid keeping up with the rapid technological developments in the world of information, communication, and media. And - if possible - it should always be a few steps ahead of business practice. There- fore, visionary and strategic discussions of our own research programs should be conducted. Preserving the close contact and increasing cooperation projects with business practice will keep us down to earth. It will become increasingly necessary to follow the already apparent specialization of the departments and institutions. All in all, challenges concerning more than one application system will probably gain importance: effectiveness and efficiency of organizations will continue to take center stage. Side conditions, as emerge from dependable architectures subject to constant improvement, have to receive increased attention. This concerns their increased immunity to infections, threats and errors as well as their guaranteed manageability by individual users, groups, or ultimately the entire organization.

Therefore, I would like to remind the editors conceptually working for WIRTSCHAFTSINFORMATIK / Business \& Information Systems Engineering and the reviewers of individual contributions that BISE primarily holds a designoriented momentum. Thus its aim should be to fulfill the principle of "research by development" and not only to offer meticulous empirical findings or constructive mathematical derivations.

BISE: Which developments in the field of information and communication systems did you expect during your time as editor-in-chief? Which were the most positive and negative surprises?

Mertens: I expected enormous progress concerning the diminishing price/performance ratio of hardware that enabled more elegant application systems.

Undoubtedly, a positive surprise was the end of the Cold War in the first place. The resulting doubling of the global capacity of work forces with a steady capital stock caused the so-called offshoring and nearsourcing, which brought new challenges to German BISE. Moreover, I did not expect the powerful and fast development of the Internet to this extent.

As a negative surprise I would consider the fact that the development of our discipline in research and business practice is not sufficiently sustainable or cumulative, and thus does not proceed efficiently. One example is the chain "internal documentation $\rightarrow$ information retrieval $\rightarrow$ selective dissemination of information $\rightarrow$ knowledge management", another the seesaw changes between automatic facto- 
ries, CIM, MES, etc., and again another the confusion regarding terms such as BI, DSS, MIS, MSS,...

Scientific results are taken up too late by business practice. Usually, new opportunities are only utilized when scientists are already done with them.

Further disappointments result from the fact that scheduling systems receive too little emphasis as an essential element of automation; collaboration between BISEand OR-experts does only succeed at few universities. Therefore, decisions of low or medium importance have to be made by people instead of having a machine calculate suitable solutions. Other sub-disciplines of applied computer science do not have to deal with this issue anymore. For instance, think of the calculation of artificial hip joints during surgeries or alternative routes in navigation devices immediately after a traffic jam warning.

The connection between BISE and industrial production or process automation would be of great interest, but only few things happen. Some people falsely think of the production site Germany as being lost. These augurs might be forced to correct themselves one day.

Finally, there are hardly any papers connecting BISE with economics or social politics. For instance, one should think much more about what a balanced path of Germany's economic growth may look like facing growing automation, a scarce number of automation experts, and a changing age structure. Together with that it would be necessary to analyze what kind of changes within project organization and system development methods would be reasonable to tap the potential of the comparatively few IT-specialists as well as that of less qualified people.

I also wonder if BISE may be able to do more in order to reduce the scandalous information gaps of executive management members, including advisory boards, e. g. as regards computer supported quantitative risk information.

BISE: Which were the most important decisions or changes for the WIRTSCHAFTSINFORMATIK journal during your time as editor-in-chief?

Mertens: I was in the position to take over a "well-organized office" where evolution and not revolution was indicated. Even more than my predecessors I tried to closely connect teaching, practice, and science as well as to foster mutual transfer. In other words: We attempted to fill the gap between articles for researchers by researchers on the one hand and the socalled little appetizers in the "glossy journals" on the other hand. Therefore, the sections "Read for you", "Surfed for You", "Discussion", "Catchword", "State of the Art”, "Students' Corner", "Innovative Product", and "Comparative Literature Study" were introduced. The common basic idea is to draw the non-specialized readers' attention to things worth reading in a directed and professional manner.

BISE: How do you assess the developments and changes of that time from a present-day perspective?

Mertens: The people in charge, with Professor Buhl as front man, are taking bold measures. I hope they will be successful. However, I am not quite sure if the former development was better than the journal's current segmentation into two versions for business practice and science, which was also discussed by the editorial board at that time. We cannot exclude the possibility that BISE will disintegrate into two "camps", as it happened with business administration in the 1960s and 70s. At that time, scientists intensively discussed type $\mathrm{A}, \mathrm{B}$, and $\mathrm{C}$ production functions without teaching their students what parts lists or PPS systems are. Or think of mathematicians who used to refuse to establish fields like financial theory or insurance mathematics at universities. Today, they recruit highschool graduates by referring to these occupational fields.

BISE: Which will be the big challenges of BISE as a scientific discipline in the next years or decades?

Mertens: In my opinion there are three major challenges:

a) Enterprises in German-speaking countries must be provided with innovative contributions at the right time (neither too early nor too late).

b) Even more than in the past, we live in a world where doctors' organizations compete for graduates in medicine, engineers' consortia compete for junior engineers, and pedagogues' associations compete for junior teachers, etc. In short: the "war for talents". At the same time, being a professor lost much of its former attractiveness due to a considerable amount of wrong political decisions. This in mind, it is absurd that science gives wrong incentives within an administrative organization of its own authority and that it establishes processes compared to which

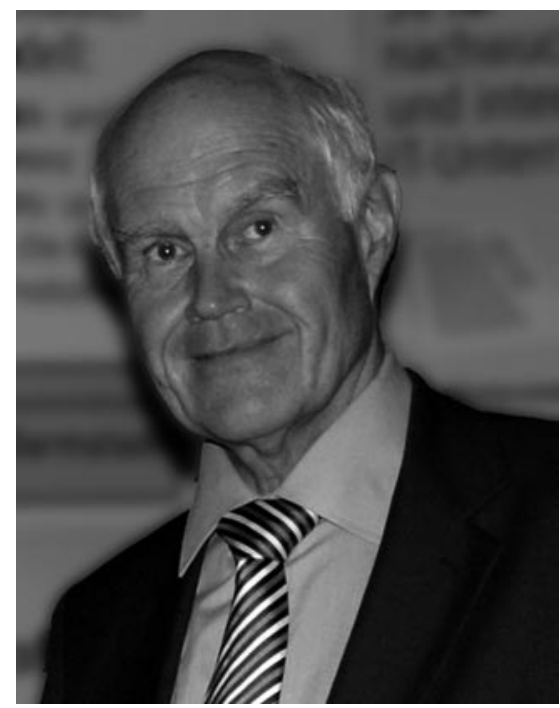

Professor Dr. Dr.h. c. Peter Mertens

Editor-in-chief from 1990 to 2000 University of Erlangen-Nürnberg mertens@wiso.uni-erlangen.de

Brussels' bureaucracy shapes up as an efficient service enterprise. As regards leading scientific journals, junior scientists must be saved from having to "optimize" their manuscripts until these fit into the usually very small intersection of three reviews and a tight page limit. Currently, reviewers can "shoot from the darkness of anonymity" and are not liable for any harm they cause. I still respectfully remember the time when eminent authorities such as Erich Gutenberg, Wilhelm Hasenack, or Karl Hax tried hard to "mature" my first works for the Journal of Business Economics, Betriebliche Forschung und Praxis, or Schmalenbach Business Review. This process was accompanied by a lot of encouragement instead of cutting criticism. Current reviewing practice should be subject to review, including time and cost reduction. However, simple solutions do not come to my mind.

c) The relation between German-language BISE and Information Systems (IS) in the U.S. should be aligned continuously using a certain amount of self-confidence. I hope that the financial crisis, which has also mainly been caused by U.S.-American scientists, especially makes those think twice who uncritically take over everything that originates from Anglo-Saxon countries. For me it seems more promising 
to learn from medicine and engineering sciences as regards research and publication practice as well as transfer.

BISE: Which developments in the field of information and communication systems did you expect during your time as editor-in-chief? Which were the most positive and negative surprises?

König: The question on which developments in the field of information and communication systems could have been expected from a 1998 perspective can hardly be answered because the years back then and now may well be called turbulent. With this restriction I would like to emphasize the term "networking" as central to the end of the 1990s. Already ten years ago the focus was no longer on technical networking, which was considered largely explored and not of interest for research any more. Instead, the focus was clearly set on "thematic networks", i. e. connections of actors via the Internet who collectively develop pharmaceuticals, construct automobiles across complex value added chains, and bundle market power against providers within procurement networks.

For me, a positive surprise during the last ten years has been Wikipedia. This general glossary, which concisely explains various terms in multiple languages and continuously as well as sustainably improves the quality of statements by a systematic usage of the "Wisdom of the Crowds", has considerably surpassed my expectations. In contrast, I have been and still am comparatively disappointed of the long time it took and partly still takes to transfer our working environment to the World Wide Web. Remember e.g. that companies prohibited their employees with Internet access to surf the Web. Actually, even among professors the regular exchange of e-mails was not yet a matter of course in 1998.

BISE: Which were the most important decisions or changes for the WIRTSCHAFTSINFORMATIK journal during your time as editor-in-chief?

König: The two major changes during my term were the implementation of the double-blind review process - which was first carried out completely in 1998 after the editorial board's decision in 1997 - as well as the journal's acceptance to the ISIdatabase so that it now regularly receives an impact factor as an important measure of reputation.
The double-blind review process, which has long been a standard procedure of many journals in international competition, means that neither the reviewer knows the author nor the author knows the reviewer. In the same step the standard of multiple revisions was introduced where reviewers and editor-in-chief take on the role of coaches. As a consequence, article quality improved significantly, especially as regards theoretical foundation and methodological approach. In the Anglo-American area this is called "rigor". In an ideal-typical natural scientific sense, this means that a reader can rebuild and reproduce an article's experimental design in his local environment such that he gets the same results as the author. For WIRTSCHAFTSINFORMATIK a particular challenge emerged because its readership - which is numerous compared to other scientific journals - is made up of practitioners by approx. two thirds. Therefore, besides rigor, published articles have to bear relevance - i. e. empirical and practical confirmation and usability of theoretical constructs in the field of application. This high standard in both dimensions made the rejection rate grow stepwise up to approx. 75-80\% (see also Fig. 5 in the article by Hasenkamp and Stahlknecht in this issue) and thus to an occasional deficit of publishable contributions - now and then.

The regime of double-blind reviewing is a prerequisite in order to be accepted for the ISI-database. The North American company Thomson Reuters (called Thomson Scientific before April 2008) runs a kind of worldwide counting service. By reporting the number of citations by other articles, an index shows an article's impact on scientific discussion. Among several thousands of journals evaluated regularly there are only a few non-English-language journals. On the one hand, this impact factor can be assigned to single authors. For instance, there are universities that base their appointment decisions on this criterion in the field of economics. On the other hand, the impact factor can be calculated for an entire journal. Today, authors still infrequently consider this criterion when deciding which journal should publish their latest research results. However, it will probably be of more relevance in future. In this context it may be interesting that the Bucconi University in Milan bases at least the younger professors' salaries on the "published impact points" to essential

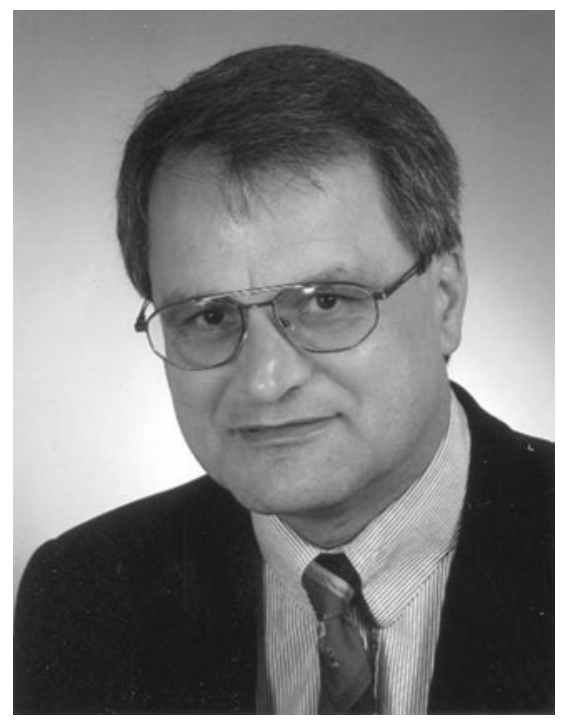

Professor Dr. Wolfgang König

Editor-in-chief from 1998 to 2008

University of Frankfurt a.M.

koenig@wiwi.uni-frankfurt.de

parts. WIRTSCHAFTSINFORMATIK is the community's only German-language journal for which an impact factor is available. Unfortunately, the expectation that the number of English-language submissions would rise due to reporting an impact factor was not fulfilled. The journal's upcoming internationalization strategy is a much more substantial attempt towards this direction.

BISE: How do you assess the developments and changes of that time from a present-day perspective?

König: In my opinion, the largest progress has been made with respect to the journal's massively improved standing within the scientific community. For instance, it performed excellently in both JOURQUAL rankings of the German Association of Business Administration Professors (Verband der Hochschullehrer für Betriebswirtschaft e. V., VHB) where more than 1,600 journals were evaluated. While belonging to the first four Germanlanguage journals in JOURQUAL1, it was even identified as the best German-language journal in JOURQUAL2 and thus surpassed all classical journals of business administration as well as a number of international journals. It can be seen that English-language journals are also ranked higher in the German-speaking community - which again is an argument for the journal's internationalization strategy. In the Peffers evaluation from 2003 (Peffers 
and Tang 2003), WIRTSCHAFTSINFORMATIK is the only journal in a language other than German ranked at position 32 of approx. 50 journals. This again is an excellent result but also shows that English-language journals simply do better due to their larger scope and higher international competition.

Another problem is the lacking ranking situation of our mother discipline computer science. While, as already mentioned, impact factors play a major role in economics, people from business administration tend to focus on the less ambitious quality measure "number of publications in A+ and A journals". Those top BISE researchers who see a certain emphasis of the field's cultural identity on business administration tend to consider this quality measure, too. As long as the focus is rather set on the culture of computer science, our community also suffers from the generally observable phenomenon that the agreement on a communitywide or even worldwide accepted ranking list is far less developed than in business administration - not to mention the even stricter impact culture of the economists. Many computer scientists emphasize the value of conferences with double-blind reviewed contributions, which they believe higher than that of journals. Clearly, conferences have been scheduled in advance so that published contributions generally cannot be enhanced throughout multiple rounds. In contrast, journals' review and revision processes generally require a higher cycle time and there is often more than one round. This cultural divergence of the BISE community became manifest e. g. in the long discussion process until a ranking list of journals and conferences was adopted in the course of the WI conference 2007 in Karlsruhe. The corresponding draft had been "softened" noticeably to make it acceptable to the majority. Still, after an impassionate discussion it was accepted finally with great majority.

BISE: Which will be the big challenges of BISE as a scientific discipline in the next years or decades?

König: We must be aware that e. g. the German Research Foundation (Deutsche Forschungsgemeinschaft, DFG) today demands and will increasingly demand BISE researchers to adopt international quality criteria - today "A+ and A journal publications" are satisfying, but the impact factor analysis lies ahead. We have to face these challenges. And in the course of today's "A+ and A journal publications" internationally acknowledged ranking lists are increasingly demanded; referring to the previously mentioned WI 2007 decision will become increasingly insufficient. For instance, this is unavoidable if we want to succeed in the next Excellence Initiative by the German federal and state governments or in the DFG's Special Research Program. In the course of this internationally quality-oriented and standardized measurable improvement, our flagship journal WIRTSCHAFTSINFORMATIK / Business \& Information Systems Engineering has to support the community and lead it on the right track. In particular, the resulting challenge is that outstanding researchers in German-speaking countries might indeed tend to publish their excellent research results - and thereby go the whole nine yards - in international top journals with higher impact factors - despite the fact that we make all contributions available both in German and English in WIRTSCHAFTSINFORMATIK / Business \& Information Systems Engineering.

Finally, I would like to point out another of our journal's substantial challenges at least in its present self-conception. The WIRTSCHAFTSINFORMATIK journal as well as the other 49 global top journals of the Peffers ranking are based on their authors' willingness to extensively - or more moderately in Germany - convey their copyrights to the publishers in the US and UK. This strategy of heckling against scientists who are supposed to develop new knowledge and of course have to assure the solid prior knowledge of their colleagues, is flanked by the fact that a "respectable journal" requires submissions to make a perceptibly different contribution in knowledge compared to any contribution published whenever and wherever. Thus, if a contribution in the same form can already be downloaded in the Internet, it should no more be published in any journal. This leads to the fact that scientists who have to hand over their copyrights have to expensively buy journals to consume their colleagues' research results - which will become even more expensive. How long will researchers continue to tolerate this?

Bernius and Hanauske (2007) give an account of the globally cultivated culture of physicists and mathematicians to virtually place all contributions on the so- called ArXiv-server in the Internet first so that every interested person can access them immediately. Generally, this happens simultaneously to a journal submission - and thus not after the publication and the consequently long time necessary for review and revision. The reader has to evaluate the contribution on his own at the beginning of its lifecycle. But he may inspire the author with his comments to improve the contribution. To what extent will quality assurance, e. g. according to the Wikipedia rules, replace the traditional review process? And in fact, why would one then need a - comparably expensive - printed version?

However, the major challenge of the BISE community in future will be: We need a higher number of first-class submissions presenting exiting and methodically well-founded research results. Without these research results and their excellent presentation, all of the previously mentioned measures constitute mere maculature.

Congratulations to the journal's $50^{\text {th }}$ anniversary. I wish the journal to continue successfully on its determined way.

\section{References}

Peffers K, Tang Y (2003) Identifying and evaluating the universe of outlets for information systems research: ranking the journals. The Journal of Information Technology Theory and Application (JITTA) 5(1):63-84

Bernius S, Hanauske M (2007) Open access. WIRTSCHAFTSINFORMATIK 49(6):456-459 